\title{
Décollement tourmant dans les pompes et les compresseurs
}

\section{Rotational flow separation in pumps and compressors}

\section{Jacques Chauvin}

Professeur,

Institut de Mécanique des Fluides, Marseille

\section{Pascal Ferrand}

Ingénieur,

Institut de Mécanique des Fluides, Marseille

\section{Mete Cen}

Assistant de Recherches

au I.V.K.,

Rhodes St-Genèse

\section{Bruno Schiavello}

Ingénieur,

Worthington Pump International, Milan

\section{Introduction}

Pour pratiquement toutes les applications contem. poraines importantes, les compresseurs et les pompes doivent opérer dans une large plage de débit et de pression de refoulement avec des rendements acceptables dans tout le domaine d'intérêt, et une durée de vie imposée. Le niveau des effets instationnaires dans l'écoulement doit être suffisamment faible pour éviter des efforts mécaniques trop élevés et des instabilités du comportement de l'écoulement dans l'ensemble turbomachine-système de vannage-circuit récepteur. Pour les compresseurs, la zone d'opération possible est limitée pour chaque vitesse de rotation, par un couple de valeur rapport de pression-débit, celui-ci étant en général positif, définissant la ligne de pompage. Pour les pompes, l'opération à débit nul est possible, mais précédée d'une large zone à rendements décroissants, dont le début correspond à l'apparition du phénomène de prérotation.

Les informations dont on dispose à l'heure actuelle permettent de penser que dans la plupart des cas, prérotation pour les pompes, et phénomènes limitant le domaine d'opération des compresseurs, sont précédés ou accompagnés de décollement toumant. On s'attache ci-dessous à faire rapidement le point des connaissances actuelles en la matière, qui ne permettent pas, à l'heure actuelle, de prédire quantitativement le comportement du système au niveau du projet ; cela devrait être réalisable dans les quatre ou cinq prochaines années.

\section{Le décollement tournant dans les compresseurs} axiaux

La grande majorité des connaissances acquises en matière de décrochage tournant l'a été dans le domaine des compresseurs axiaux. Un sommaire des connaissances à différentes époques et des listes très complètes de références ont été établis notamment par la NACA [1], Yershov [2], Fabri [3] et Orner [4]. Ce régime de décollement est marqué par l'existence de zones de débit réduit, nul ou négatif, de nombre, d'étendue circonférentielle et radiale variables, se déplaçant en moyenne pour chaque configuration comme un corps solide, à vitesse angulaire constante par rapport aux aubages. Les configurations obtenues sont fonction $\mathrm{du}$ dessin de la machine, de sa vitesse de rotation et du débit moyen aux conditions nominales. Le nombre de cellules peut varier de 1 à 8 et plus, la vitesse de rotation par rapport à celle de la roue, de 0.2 à 0.8 environ, mais est en général voisine de 0.5 . On distingue deux types de "décollement" : le décollement faible, qui n'affecte pas ou peu les caractéristiques globales de la machine, ou des étages directement concemés, et le décollement fort, en général à petit nombre de cellules, qui modifie profondément la caractéristique pression-débit de l'étage du compresseur où il prend naissance, et modifie la forme de la courbe de performance globale. Le premier peut entraîner la rupture des aubages par fatigue, le second conduit, à fortiori, à des sollicitations mécaniques et thermiques élevées et, suivant la réponse de l'ensemble du sytème compresseur-circuit (Greitzer [5]) est l'un des mécanismes permettant de conduire au "pompage", c'est-à-dire à une opération à débit variable dans le temps, avec une fréquence faible mais des amplitudes considérables. considérables.

\section{Description physique du phénomène}

On présente souvent le décrochage tournant comme dû au décrochage au sens stationnaire à un rayon donné, 


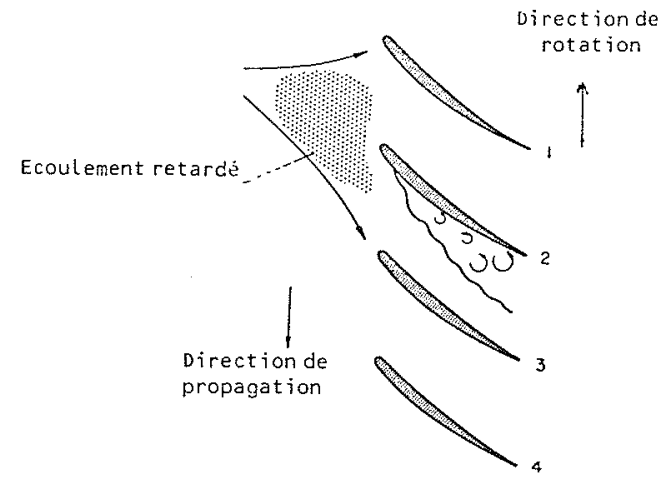

Figure 1 - Décrochage tournant

d'un ou de plusieurs aubages sous l'effet d'une petite perturbation localisée. Le blocage produit par ce décollement forcerait le débit correspondant à ce ou ces passages, à dévier de part et d'autre de la zone bloquée, augmentant l'incidence sur les aubages situés au-dessous de la zone (fig. 1) et diminuerait pour ceux situés audessus, entraînant le décollement des premières et le recollement pour les secondes. Cette explication physique ne tient pas car elle supposerait, entre autres, un "saut" discret aube par aube alors que la zone à débit réduit progresse de manière continue.

On peut en retenir trois éléments : une petite perturbation localisée voit ses effets amplifiés; il y a passage d'un régime stable, mais à la limite de sa stabilité (régime sans décollement tournant) à un autre régime stable (avec décollement tournant); l'apparition de régions à débits réduits est facilitée par la proximité du décollement statique, c'est-à-dire pour des vitesses débitantes pour lesquelles les gradients de pertes d'aubage ou de paroi et de la déviation en fonction de l'incidence ou du Mach sont importants.

Fabri [3] a montré, pour des cas simples, mais tenant compte des pertes, que l'apparition du décrochage tournant dans une rangée d'aubes était liée à la possibilité, pour les cellules à débit réduit et une partie de l'écoulement sain de tourner comme un corps solide, et était donc liée à la forme de l'écoulement tourbillonnaire engendré par les aubages et au débit, de faibles débits ne permettant pas de remplir toute la veine en satisfaisant à l'équilibre radial. Il a mis en évidence le fait que le décrochage toumant ne dépendait pas directement du décrochage stationnaire des profils. De nouveau 4 éléments sont à retenir : l'influence de la vorticité de l'écoulement ; la nécessité de satisfaire l'équilibre radial, même en régime décroché ; la condition nécessaire mais non suffisante de possibilité de rotation des zones à écoulement réduit en corps solides ; le fait que la présence des zones à débit réduit est nécessaire pour que, en zone saine, l'équilibre radial soit satisfait.

Yershov [2], sur la base de nombreux résultats expérimentaux, constate qu'en dehors des zones à débit réduit, les conditions d'écoulement correspondent approximativement à celles qui donnent le rapport de pression maximum quand il n'y a pas décrochage tournant, pour le petit décrochage et pour le grand décrochage, à celles qui correspondent au flux d'énergie maximum.
Ces considérations justifient le fait que l'étendue globale de la zone à débit réduit croisse lorsqu'on réduit le débit, mais ne donnent aucune indication sur les paramètres déterminant le nombre de zones et leur vitesse de rotation.

Les études plus récentes de Day et Cumpsty [6] indiquent que l'approche "compresseurs parallèles" peut être suivie, ce qui conduit à des conditions hors cellules à débit voisin de celui indiqué par Yershov, pour le grand décrochage.

Expérimentalement, on constate que le blocage nécessaire peut être réalisé par blocage (décollement) annulaire sur $360^{\circ}$, petit décrochage ou grand décrochage.

\section{Effets des rangées d'aubes voisines}

Les configurations (nombre de zones et vitesse de propagation) ne sont pas les mêmes pour un compresseur monoétage et pour un compresseur multiétage composé des mêmes aubes, d'après la NACA, ce qui est confirmé par [6] mais, qualitativement, les phénomènes sont les mêmes.

Lorsque les perturbations sont transmises à travers le compresseur, leur mode de propagation est particulier : elles ne suivent pas l'évolution circonférentielle de l'écoulement sain, mais se propagent quasi axialement, fait mentionné par la NACA avant 1956, et confirmé par [6] en 1977. Dans ce cas, du fluide émanant des zones saines doit traverser circonférentiellement les zones décollées, principalement à l'amont des rotors, induisant une prérotation (fig. 2).

L'expérience montre encore que le grand décollement toumant correspond le plus souvent à une configuration à une cellule, couvrant toute la hauteur de la

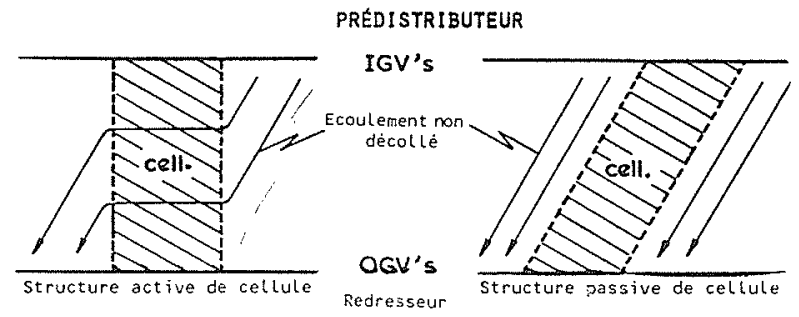

Figure $2 \mathrm{a}$ - Comparaison de structure de cellule active et passive (extraite de la Réf. [6]).

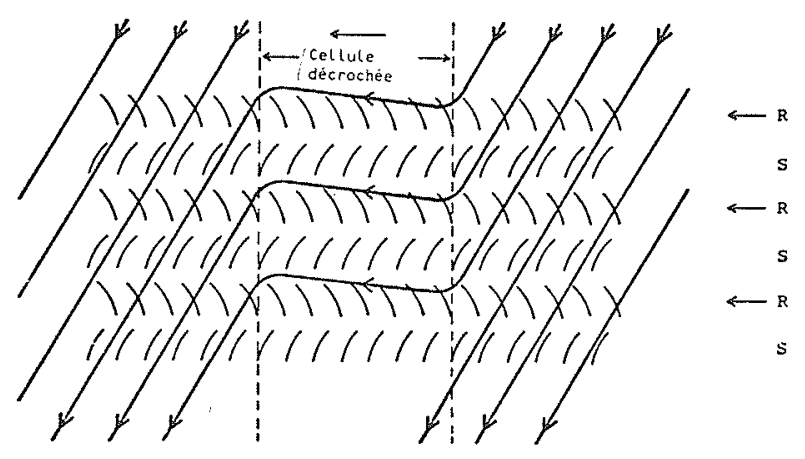

Figure $2 b-$ (extraite de la Réf. [6]). 
veine, et d'étendue circonférentielle importante, avec écoulements locaux de retour importants.

L'expérience et un certain nombre de calculs par des méthodes relativement sophistiquées montrent que si les phénomènes sont tridimensionnels, une approche simplifiée peut suffire pour représenter correctement leurs caractéristiques essentielles, du moins pour le petit décrochage. Omer [4], par exemple, prend une approche tranche par tranche sur la hauteur de l'aube selon des surfaces de courant définies pour l'écoulement sans décrochage et utilise comme couplage radial la condition nécessaire de rotation moyenne en corps solide des zones de débit réduit. Ces phénomènes sont de caractère fortement non linéaire.

Schémas possibles pour l'établissement de méthodes de prévision du décrochage tournant et de ses conséquences

On admet que le décrochage tournant est un régime stable, qui ne peut être atteint, au départ du régime d'opération normal, qu'en passant par un régime instable ou transitionnel.

Toute méthode de calcul doit donc permettre la détection de l'apparition de l'instabilité. Cependant, celle-ci peut conduire à un régime stable caractérisé par un décrochage annulaire $\left(360^{\circ}\right)$ affectant une partie de la hauteur d'aube, à un décrochage tournant faible ou un décrochage tournant fort. La possibilité de détecter la forme du deuxième régime stable ou des suivants, s'il y a séquence, doit être incorporée dans le calcul, en tenant compte de l'environnement du compresseur complet.

Ces différents régimes sont déterminés essentiellement par les caractéristiques aérodynamiques du compresseur. Leurs conséquences -apparition du pompage ou non par exemple-, sont fonction de la géométrie d'ensemble du circuit (Greitzer [5] par exemple), dont on devra tenir compte.

Une méthode complète devrait permettre de calculer le nombre de cellules, leur vitesse de propagation et leur étendue radiale et azimutale, en fonction du point d'opération (couple débit-pression), que celui-ci soit atteint par un vannage variable à vitesse de rotation constante, ou, de manière plus réaliste, par vannage constant à vitesse variable. Le temps normal d'établissement des régimes et les phénomènes d'hystéresis rendent le mode d'opération dépendant de la manière suivie pour l'atteindre.

\section{Méthode détaillée}

Orner [4] a jeté les bases d'une méthode de ce type, qui constitue en fait une généralisation de la méthode non linéaire de Takata et Nagano [7], aux écoulements en compresseurs mono ou multi-étages. La méthode suppose la connaissance de l'écoulement en l'absence $\mathrm{du}$ décollement toumant (écoulement stationnaire axisymétrique). Une perturbation instationnaire d'amplitude et de fréquence quelconque est alors superposée à l'écoulement de base et son évolution est étudiée surface de courant par surface de courant, les équations du mouvement comportant comme seules hypothèses l'incompressibilité de l'écoulement de perturbation et le maintien en moyenne des surfaces de cou- rant déterminées par calcul axisymétrique. Une liaison radiale tranche par tranche est obtenue en imposant la rotation des cellules en corps solide au départ d'une section mère.

Des équations instationnaires de conservation de débit et de quantité de mouvement en direction méridienne et tangentielle, on dérive les équations principales suivantes, pour les espaces sans aubes:

$$
\begin{aligned}
\frac{\partial}{\partial t} \xi_{p} & +\left(A+W_{p m}\right) \frac{\partial}{\partial m} \xi_{p}+ \\
+ & \frac{1}{r}\left(B+W_{p} \theta\right) \frac{\partial}{\partial} \xi_{p}+\left(A+W_{p m}\right) k_{n} \xi_{p}+ \\
+ & W_{p m}\left[\frac{\partial c}{\partial m}+k_{n} c+\frac{\partial}{\partial m}(2 \omega r \sin \epsilon)\right]+ \\
& +2 \omega r \sin \epsilon\left(\frac{\partial W_{p m}}{\partial m}-\frac{1}{r} \frac{\partial W_{p m}}{\partial \sigma}\right)+D=0
\end{aligned}
$$

où $A, B, C$ et $D$ sont des valeurs connues par le calcul stationnaire, les $W_{p}$ sont les vitesses de perturbation, suivant la direction méridienne $(m)$ et tangentielle $(\theta)$, $\xi_{p}$ est la vorticité de l'écoulement perturbation ; $k_{n}$ est la courbure locale de la ligne de courant ; $r$ le rayon ; $\epsilon$ l'angle entre la normale à la surface de courant et le rayon.

Pour les espaces aubés, considérés comme des disques d'action d'épaisseur finie ( 1 amont, 2 aval)

$$
\begin{aligned}
& \frac{\partial W_{p} \theta_{1}}{\partial t}=\frac{r_{2} \rho_{2}}{r_{1} \rho_{1}} \frac{\partial}{\partial t}\left[\left(A_{2}+\eta W_{p m 1}\right) \operatorname{tg} \beta_{2}+\right. \\
& +\left(A_{1}+W_{p m 1}\right) \xi_{1}-\frac{\rho_{2} r_{2}}{\rho_{1} r_{1}}\left(A_{2}+W_{p m 1}\right) \xi_{2}+ \\
& \quad+2 \omega\left(A_{1}+W_{p m 1}\right) \sin \epsilon_{1}- \\
& -\frac{\rho_{2} r_{2}}{\rho_{1} r_{1}}\left(A_{2}+\eta W_{p m 1}\right) \sin \epsilon_{2}-\frac{1}{2}(1+\eta) \frac{c}{r_{1}} \frac{\partial^{2} W_{p m}}{\partial t \partial \theta} \\
& -\frac{1}{r_{2}} \frac{\partial}{\partial \theta}\left[\frac{1}{2} \bar{\pi}\left(A_{1}+W_{p m 1}\right)^{2}+\left(B_{1}+W_{p 1} \theta_{1}\right)^{2}\right]
\end{aligned}
$$

avec $\pi$, coefficient de perte de pression totale défini par $\bar{\pi}=\bar{\pi}_{s}+\bar{\pi}_{d}-\tau_{\pi} \frac{\partial_{\pi}}{\partial t}$ où $\bar{\pi}_{S}$ est basé sur les informations stationnaires, mais calculé pour l'angle d'entrée instantané, $\tau_{\pi}$ un terme d'inertie tenant compte de ce que $\beta_{1}$ est fonction du temps et que la couche limite n'est pas en équilibre instantanément $; \bar{\pi}_{d}$ correspond aux pertes additionnelles dans la zone à débit réduit. C'est l'ajustement de ce coefficient qui permet le synchronisme de rotation. L'angle $\beta_{2}$ est défini par

$$
\beta_{2}-\tau_{\beta} \frac{\partial \beta}{\partial t} .
$$

Les coefficients $\tau_{i}$ permettent d'introduire une hystérésis, mais à surface nulle. La relation entre les pressions totales relatives et le coefficient de perte est 
pris comme :

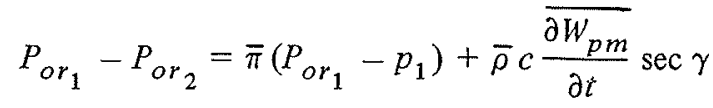

le deuxième terme du membre de droite tenant compte de manière approximative du caractère instationnaire de l'écoulement ( $c$ corde, $\gamma$ calage, $\bar{\rho}$ et $\bar{W}_{p m}$ densité et vitesse de perturbation moyenne).

Le système d'équation est résolu numériquement. Le régime final obtenu est montré être indépendant en fréquence, en vitesse de rotation et en amplitude des zones, de la perturbation donnée initialement.

Une comparaison calcul/essai est donnée en figure 3, qui montre le potentiel de la méthode pour le calcul du début du décollement, du nombre de cellules et de leur évolution, et de la vitesse de rotation dans le cas du petit décrochage ; la méthode permet aussi de prédire le décrochage annulaire. Elle ne prend pas en compte la réaction du système complet. La méthode relativement peu couteuse pour des espaces interaubes de l'ordre de la corde, permet les études paramétriques. Elle est indicative des possibilités actuelles.

\section{Méthode globale}

Il est cependant évident qu'au stade de l'avant projet, une méthode à la fois plus globale et donc plus grossière, et plus rapide, serait bienvenue. On peut penser à une utilisation séquentielle de méthodes simples de type mono dimensionnel dont les éléments de base existent.

Il faut d'abord disposer d'un outil pour déterminer la possibilité d'apparition des régimes instables permettant la transition.
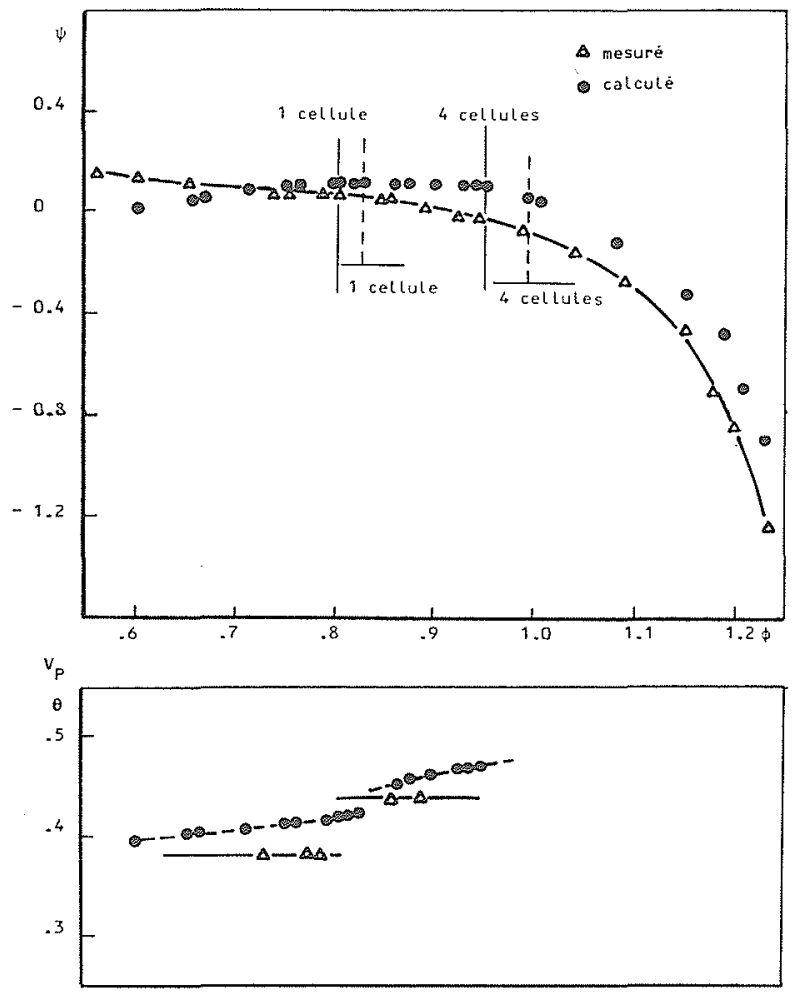

Fig. 3 - Comparaison des calculs ế des mesures pour le compresseur NACA.
L'IMFM, sur la base d'une formulation proposée par Fabri [9] pour l'étude instationnaire d'effets de distorsion, développe une méthode de calcul à la ligne moyenne pour compresseurs multiétages, tenant compte des espaces vides. On superpose à l'écoulement de base une perturbation. Celle-ci peut être de forme quelconque dans le temps et dans l'espace et avoir son origine à l'amont, à l'aval ou dans la machine.

L'étude se fait plan par plan suivant l'axe machine sur une surface de courant représentative des surfaces d'ensemble des aubages. Les grilles d'aubes sont schématisées par des disques d'action d'épaisseur nulle à fonctions de transfert connues (pertes et angles fonction de l'incidence et du Mach). Pour les espaces hors grilles, les équations d'Euler sur une surface de courant à faible variation de rayon sont utilisées

$$
\frac{\partial}{\partial t} \xi_{p}+A \frac{\partial}{\partial m} \xi_{p}+\frac{1}{\gamma} B \frac{\partial}{\partial \theta} \xi_{p}+A k_{n} \xi_{p}+D=0
$$

Les espaces aubés sont définis par des relations entre les grandeurs aérodynamiques entre l'entrée et la sortie à l'aide des coefficients suivants : $\pi, \partial \pi / \partial i, \partial \pi / \partial M$, $\partial \beta_{s} / \partial \beta_{e}$. La résolution s'effectue en utilisant, d'une part la décomposition en série de Fourier pour caractériser les dérivées partielles par rapport à la circonférence et, d'autre part, la transformée de Laplace pour les variations temporelles. Le système est résolu suivant l'axe machine. L'image par la transformée de Laplace des grandeurs caractéristiques ne présente sous la forme d'une fraction rationnelle $H(S)=\frac{N(s)}{D(s)}, H(S)$ étant définie par la transformée de Laplace

$$
X^{\prime} n(m, S)=\int_{0}^{+\infty} e^{-S t} X^{\prime} n(m, t) d t .
$$

La stabilité du système est étudiée dans un premier temps en traçant dans le plan Nyquist le point $\operatorname{Im}(D(i \omega)), \operatorname{Re}(D(i \omega))$ pour $S=i \omega$ : le nombre de solutions instables est égal au nombre de tours effectués par cette courbe autour de l'origine, lorsque $\omega$ varie entre $-\infty$ et $+\infty$. Dans une deuxième étape, le tracé de la fonction complète (fig. 4) permet de déterminer les fréquences de résonances définies par les valeurs de $\omega$ correspondant aux maxima du module de $H(i \omega)$. Ces fréquences représentent les vitesses de rotation critiques d'une perturbation qui se trouverait ainsi amplifiée.

Ensuite, en prenant $s=0$ (fin du régime transitoire), une recomposition plan par plan détermine l'amplification ou l'amortissement spatial de chaque élément. Par cette méthode on espère pouvoir détecter la sensibilité de la machine aux perturbations axiales, circonférentielles avec variation de la fréquence, c'est-à-dire cemer les zones d'instabilité correspondant à divers modes d'excitations.

L'apparition d'une instabilité étant connue, il faut déterminer le type de régime stable suivant qui s'établira : décrochage annulaire ou d'aubage ; décrochage tournant faible ou fort ; éventuellement, pompage.

Yershov [2] propose une méthode permettant, au départ de la caractéristique en écoulement axisymé- 


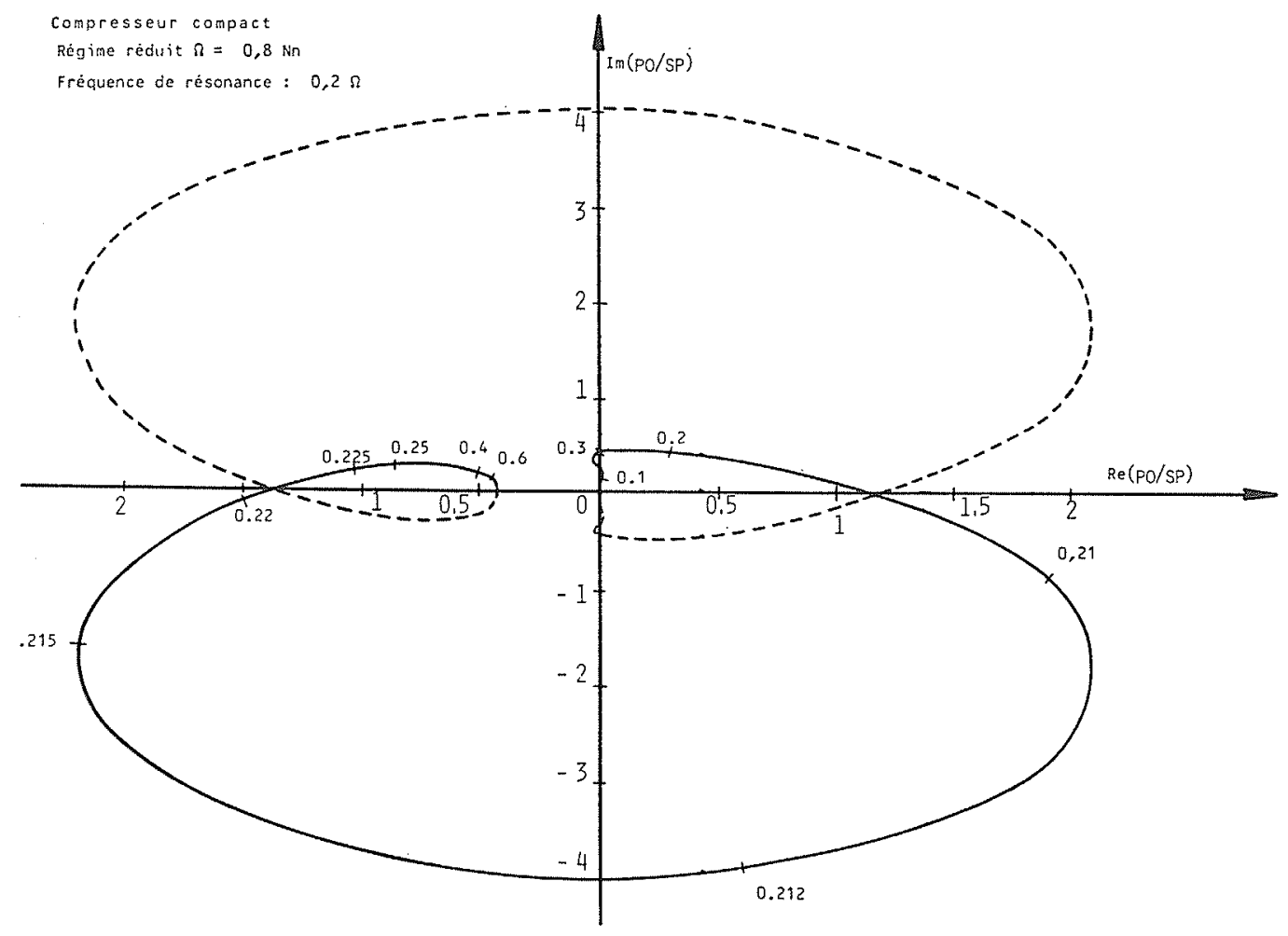

Figure 4

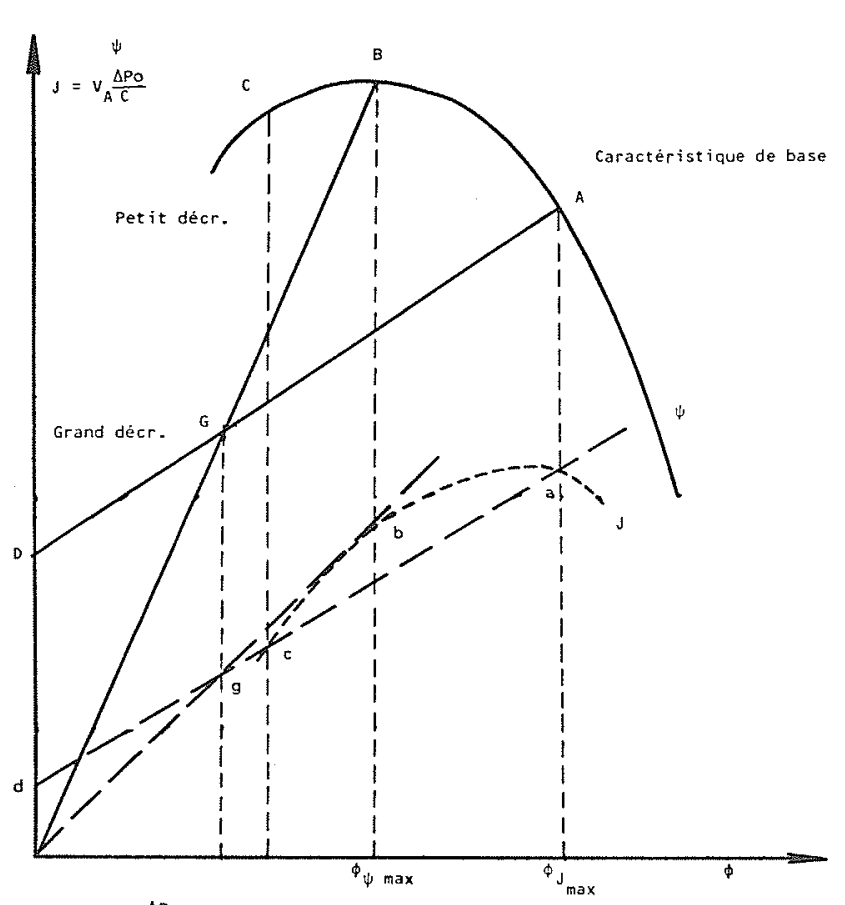

$J_{P D}=c_{A}\left(\frac{\Delta P_{O}}{C}\right)_{\text {max }}$

$J_{G D}=c_{A_{J \max }}\left(\frac{\Delta P_{0}}{C}\right)\left(1-\bar{l}_{30}\right)+0.1 \bar{l}_{30} \quad c_{A_{J_{\max }}^{3}}^{3}$

Figure 5 - Approche de Yershov. Energie maximum.

trique d'un étage de compresseur, de déterminer la branche de gauche de la caractéristique, en considérant que le mode d'opération est celui qui correspond à l'échange d'énergie maximum.

On trace au départ, la caractéristique pression/débit et la caractéristique énergie échangée $J=C_{a} \frac{\Delta p_{o}}{\rho}$ (figure 5 ; cas incompressible).

Pour les cas avec décollement tournant, on calcule en considérant qu'une partie de la section de passage (ou de la circonférence, au rayon moyen) n'est traversée par aucun débit (largeur effective de cellules $\tau_{30}$ ). L'énergie échangée est alors avec $c_{a}$ la vitesse débitante en dehors de la zone d'eau morte

$$
J=c_{a} \frac{\Delta p_{o}}{\rho}\left(1-\ell_{30}\right)+\Delta J
$$

où $\Delta J$ est l'énergie communiquée à la zone d'eau morte

$$
\Delta J \simeq\left(\bar{\ell}_{30}-\bar{\ell}_{3}\right) c_{a} \frac{c_{a}^{2}}{2}\left[1-\left(\frac{c_{a_{3}}}{c_{a}}\right)^{2}\right]
$$

avec $\bar{\ell}_{3}$ l'étendue réelle de la zone à débit réduit et $c_{a_{3}}$ la vitesse débitante moyenne sur $\bar{\ell}_{3}$.

Pour le petit décrochage, on suppose $\Delta J=0$ et, en admettant que les conditions en dehors des zones d'eau morte correspondent aux conditions de pression maximale, $c_{a *},\left(\Delta p_{o} / \rho\right)_{*}$

$$
J_{P D}=c_{a *}\left(\frac{\Delta p_{o}}{\rho}\right)_{*}\left(1-\vec{\ell}_{30}\right)=c_{a}\left(\frac{\Delta p_{o}}{\rho}\right)_{*}
$$

Yersov suppose que, à débit résultant nul, $\Delta \simeq 0$ est encore valide et que $\Delta p_{o}=0$ (ceci peut être corrigé, notamment au vu de [6]). Les points caractéristiques $p_{o}$ et $J$ à décollement faible se situent donc sur une droite qui, pour Yershov, passe par le maximum de la courbe caractéristique et l'origine. 
Pour le grand décollement, l'expérience indique, d'après [2], que l'écoulement en dehors de la zone d'eau morte est voisin de celui qui se produit pour $J=J_{\max }$ dans l'écoulement non perturbé, situé à $c_{a_{0}}>c_{a}$ * (fig. 5) ; $\Delta J$ n'est plus négligeable. Yersov supposant que $\frac{c_{a_{3}}}{c_{a_{0}}} \simeq 0.3$ (résultats d'essais) obtient finalement :

$$
\left.J_{G D}=c_{a_{0}}\left(\frac{\Delta p_{0}}{\rho}\right)\left(1-\bar{\ell}_{30}\right)+0.1 \bar{\ell}_{30}\right) c_{a_{0}}^{3}
$$

et $J$ varie encore linéairement en fonction de $c_{a}$, de même que $\left(\frac{\Delta p_{0}}{\rho}\right)_{0}$

Suivant les positions relatives des courbes $J, P_{p 0}$, $J_{G D}$, en appliquant le principe de l'énergie maximum, on peut trouver le régime d'opération le plus probable.

Ayant évalué les performances des étages, il est possible de recourir à un calcul d'empilement d'étages et de voir si l'un ou plusieurs de ces étages fonctionnent en grand décrochage pouvant entraîner le pompage, ainsi que d'indiquer les régions soumises au petit décrochage sans l'évaluer en détail.

La référence [8] présente une corrélation expérimentale basée sur un modèle assez voisin de celui de Yershov, et sur l'opération en "compresseur parallèle" pour les parties décrochées et non décrochées, mettant en valeur l'importance de l'effet de blocage de la cellule décrochée. Elle détermine les valeurs limites de celui-ci pour la transition entre régime sain, petit et grand décrochage, en tenant compte de l'hystéresis possible, à l'aide de valeurs empiriques du blocage et du coefficient de pression. Son utilisation permet de faire ressortir l'influence du coefficient de débit nominal, du nombre d'étages et de la pente de la ligne de vannage sur les performances en régime décroché.

Récemment, Greitzer [5] a mis au point une méthode permettant de distinguer, en tenant compte du circuit de décharge et du vannage, si la machine opère en pompage ou en grand décrochage et d'indiquer si le fonctionnement se stabilise en un point de caractéristique ou opère suivant un cycle limite. Le compresseur est schématisé par un disque d'action infiniment mince et un tuyau, le réservoir de décharge par un volume, la vanne par un orifice avec perte de charge et un tuyau. L'application de la méthode Greitzer nécessite la connaissance complète de la courbe caractéristique du compresseur, au moins jusqu'au débit nul. L'analyse fait ressortir que le paramètre le plus important pour le mode opératoire d'ensemble est $B=\frac{U}{2 a} \sqrt{\frac{V_{p}}{A_{c} L_{c}}}$ avec $a$ la vitesse du son, $U$ vitesse de rotation, $V_{P}$ volume du réservoir de décharge, $A_{c}$ et $L_{c}$ section et longueur du tuyau équivalent au compresseur. Si $B<0.45$ on a opération en grand décrochage. Pour $0.45<B<0.6$, on a pompage avec cycle limite, avec ou sans écoulement de retour.

Ce qui précède indique que, sans prétendre peut être à une précision suffisante, les éléments existent pour prédire le décrochage tournant et ses effets moyennant l'étalonnage de fonctions ou constantes empiriques.
Décollement dans les compresseurs et pompes centrifuges. Prérotation

Sans parler du décollement tournant constaté dans les diffuseurs sans aubes de machines centrifuges - et qui confirme l'influence primordiale de la loi de giration (ainsi qu'un phénomène analogue apparaissant en météorologie) et le peu de valeur du modèle physique "décollement sautant", - de nombreux auteurs $[2,9,10,11]$, remarques de Bammert sur [5], etc. ont constaté l'exis. tence de décollements tournants dans les rouets de machines centrifuges, en général à 1 ou 2 cellules, d'étendue radiale partielle d'abord, puis totale, à l'entrée et avec des vitesses de rotation de 0.13 à $1.0 u$, les roues étant suivies de volute. diffuseurs sans aubes ou diffuseurs aubés. L'existence de caractéristiques discontinues, associable à un décollement tournant fort, est bien connue.

Les résultats expérimentaux sont assez fragmentaires. La détection du phénomène est opérée en amont des roues. Aucune approche théorique spécifique n'est signalée. Il serait bon de voir si les méthodes valables pour les compresseurs axiaux sont susceptibles d'être appliquées aux compresseurs centrifuges.

En ce qui concerne les pompes, deux cas précis ont été signalés dans les références [12] et [13]. Le décrochage tournant ne parait pas influencer fortement les caractéristiques. [14] attribue la différence de comportement entre compresseurs et pompe aux effets de compressibilité. Les références $[9,10,13$ et 14] indiquent que la prérotation d'ensemble de l'écoulement d'entrée à une vitesse voisine de celle de rotation de la roue, est précédée d'une phase de perturbation due au décrochage tournant, à vitesse voisine de 0.5 de celle de la roue; l'effet d'asymétrie de la volute ne paraissant pas influencer le phénomène de décrochage toumant [13] a également montré que pour la génération de la prérotation, l'effet de volute était secondaire devant celui dû au dessin de la roue et, en particulier, à l'apparition de conditions voisines de celles du décollement statique au bord d'attaque de l'aube en tête.

La détection du décrochage toumant permettrait donc de servir comme avertisseur de prérotation; il indique les premiers écoulements de retour. Cependant, pour les pompes, ce décrochage ne persiste que sur un intervalle étroit de débit, étant rapidement masqué ou supplanté par l'apparition de cavitation locale dans les régions décollées, juste avant la prérotation.

\section{Références}

[1] N.A.S.A.

[2] YERSHOV V.N. - Unstable conditions of Turbodynamics rotating stall FTD-MT-24-04-71. Foreign Technology Air Force Systems Command 1971 Traduction d'un ouvrage Russe de 1966.

[3] FABRI J. - Rotating stall in axial flow compressors. Dans Unsteady Flows in axial Flow Compressors. V.K.I. L.S. 20-1970.

[4] ORNER N. - Prédiction du décrochage tournant dans les compresseurs axiaux. Thèse de Doctorat. Faculté Polytechnique de Mons et Institut Von Karman, Mai 1976.

[5] GREITZER E.M. - Surge and Rotating Stall in axial Flow Compressors. Part I and II. Trans. A.S.M.E. Journal of Engineering for Power, April 1976, pp. 191-217. 
[6] DAY I.J. et CUMPUSTY N.A. - The Measurement and interpretation of flow rotating stall cells in axial com. pressors. Univ. of Cambridge. Dept. of Engineering, CUED/ A. TURBO/TR90-1977.

[7] TAKATA $H$. and NAGANO S. - Non linear analysis of rotating stall. A.S.M.E., Paper n ${ }^{\circ} 72-65-3,1972$.

[8] DAY I.J. GREITZER E.M. and CUMPSTY N.A. - Prediction of compressor performance in rotating stall. A.S.M.E. Jour. of Eng. for Power, Vol. 100, Jan. 1972.

[9] MUZUKI S. - Investigation concerning rotating stall and surge phenomena within centrifugal compressor channels. A.S.M.E. Public., Vol. 19, n 135 , Sept. 1976.
[10] KUBO T. and MURATA S. - Unsteady flow phenomena in centrifugal fans (Rotating flow patterns in inlet ducts). Bull.J.S.M.E., Vol. 19, $\mathrm{n}^{\circ} 135$, Sept. 1976.

[11] SELENEV K.P. et al. - Machine énergétique, $n^{\circ}$ 3, (19693), p. 20 et suivantes (en russe).

[12] SAZONOV A.A. - Turbomachines et écoulements, $\mathrm{n}^{\circ} 6$, (1972), p. 151 et suivantes (en russe).

[13] JANIGRO A. et SCHIAVELlO B. - Prerotation in centrifugal pumps. Design criteria. Dans Off Design Performance of Pumps. VKI L.S. 78-3. Feb. 1978.

[14] CEN M., SCHIAVELLO B. - Communications privées.

\section{Discussion}

M. le Président. - Je remercie M. CHAUVIN de cet exposé sur l'aspect spécifique de l'écoulement tournant et sur les méthodes actuelles de prédiction et de calcul. Pour moi, ce qui est nouveau aujourd'hui, c'est que je n'avais pas encore entendu parler de petits et de grands décrochements. A l'occasion, il faudra que nous en discutions ; il y a quelque chose qui m'étonne un peu.

M. GUITON. - Je tiens à remercier M. le Professeur CHAUVIN d'avoir bien voulu prendre sur son temps actuellement très chargé pour nous présenter l'essentiel de ce qu'on sait sur les décollements tournants. Ceci rentre dans le cadre du Groupe de Travail $\mathrm{N}^{\circ} 1$ de la Division "Applications Industrielles de la Mécanique des Fluides" de la SHF, Groupe de Travail qui se consacre à l'étude du fonctionnement des turbomachines à débit partiel.

M. CHAUVIN et ses co-auteurs ont tenté, je crois, d'établir un pont entre ce qui se passe dans les compresseurs et ce qui se passe dans les pompes pour lesquelles on parle plus souvent de "prérotation" que de décollement tournant.

J'ai noté au début de l'exposé que l'un ou l'autre des deux types d'instabilité était favorisé suivant que le rotor avait un "débit nominal élevé" ou un "débit nominal faible", le décollement pariétal que l'on appelle communément "la prérotation" tendant à apparaître dans ce dernier cas. C'est celui des pompes qui ont des aubages beaucoup moins chargés que les compresseurs.

La différence de géométrie entre pompes et compresseurs joue donc, probablement, un rôle déterminant.

M. CASACCI. - Le problème est bien celui que M. GUITON vient de résumer. Il semble que votre méthode pour les compresseurs soit celle d'une prédiction globale, d'une approche globale.

La question qu'on pourrait vous poser est celle-ci : avez-vous étudié un peu finement le problème du changement de structure de l'écoulement dans une aube ou dans un système d'aubage lors qu'on s'éloigne du point de meilleur tendement ? est-ce que dans ces changements de structure de l'écoulement il n'y a pas des idées à trouver pour les projeter dans l'étude globale ?...

Par exemple, on parle du côté "dépression", puis on fait des calculs ; on a les conditions à l'amont et les conditions à l'aval ; on pense que tout se passe bien. Mais si les conditions, côté dépression et côté pression, sont complètement différentes, et si les deux écoulements se croisent à la sortie, tout ce que vous avez calculé ne sert plus à rien.

Autrement dit, est-ce qu'il pourrait y avoir une étude un peu fine du changement de structure dans un cas relativement simple ?... Il faut attaquer les problèmes des deux côté, bien sûr : il faut une réponse globale, parce qu'on en a besoin rapi- dement ; mais il faut aussi des études un peu fondamentales pour comprendre la physique du phénomène

Dans les turbines hydrauliques Kaplan, quand on est à l'emballement on sait qu'il y a une partie des aubes qui fonctionne en turbine et une partie qui fonctionne en pompe, si bien que l'on consomme de la puissance pour rien. On atteint un débit qui est parfois le double du débit normal. Le débit aval de la machine est très grand : il devient deux fois le débit nominal. Il faut bien que l'énergie se dissipe quelque part.

Est-ce que l'étude de ce changement de structure n'est pas assez fondamental?

M. CHAUVIN - Si. Un peu rapidement j'ai donné un certain nombre de réponses. La première chose, c'est que juste avant que le pompage ou le décrochage ne se produise on pensait que de profondes modifications de la distribution des filets fluides apparaîtraient. Sur un certain nombre de compresseurs, on a constaté que ce n'était pas le cas.

D'un point de vue global, ce qui se produit et qui paraît déterminant, ce sont des changements de pertes et des déviations ou des facteurs de glissement.

Pour les compresseurs axiaux on constate, comme pour la turbine Kaplan, un phénomène de recirculation important pour le grand décrochage.

En décrochage faible, on a pu constater que la recirculation n'occupait qu'une partie de la hauteur d'aube et que le débit de retour et donc le débit total étaient presque nuls dans cette région. Le fluide des zones décrochées tourne avec la roue mais le reste de l'écoulement est un écoulement normal.

Quand on passe à une instabilité plus profonde, celle qui conduit à la caractéristique debit-pression stylisée par une horizontale, des écoulements de retour localisés importants apparaissent, la partie saine opérant aux conditions correspondant à un échange d'énergie maximum.

Il est important de connaître le détail de l'écoulement pour bien comprendre le phénomène, mais déjà il apparaît qu'avec des hypothèses globales on peut prédire les fréquences de décrochage.

M. CASACCI. - D'où ces calculs qu'on schématise un peu. C'est en fait plus ou moins asymétrique.

M. CHAUVIN. - La méthode actuelle tient compte de l'asymétrie des écoulements.

M. LE BOT. - Peut-on espérer tenir compte dans les modèles de calcul en cours de développement de l'influence des traitements de carter sur la ligne de décrochage des compresseurs axiaux ?

M. CHAUVIN. - La seule façon dont nous l'ayons fait, c'est 
sur l'écoulement de base : on fait des corrections de blocage et de pertes d'extrémité. Mais, dans le calcul de la cellule ellemême, on n'en tient pas compte. C'est simplement par une distribution de pertes, ou un niveau de pertes et un gradient de pertes qu'on en tient compte.

Je ne crois pas qu'on ait très bien compris ce qui se passait au niveau des effets de paroi.

M. LE BOT. - Comment peut-on appliquer les méthodes de calcul des "compresseurs parallèles" en régime de décollement tournant?

L'influence de la distance entre aubages fixes et aubages mobiles a-telle été étudiée expérimentalement pour préciser la structure des "cellules actives" du décollement tournant

L'expérimentation d'un compresseur monoétage a montré qu'un distributeur à aubes ne présentant pas de déviation et à corde importante placé très près du totor aval, peut empêcher l'organisation du décollement tournant. Qu'en pensez-vous?

M. CHA UVIN. - Il y a un changement radical dès qu'on met un obstacle amont. La présence d'un simple obstacle modifie complètement la configuration et cela peut aller jusqu'à soit empêcher l'apparition du phénomène, soit aussi le stabiliser complètement.

M. le Président. - Comme il semble que pas mal de personnes ne sont pas très au courant de ces problèmes de décollement toumant, je voudrais signaler que le document publie par le groupe de M. GUITON comporte de nombreuses informations à ce sujet.

D'autre part, à l'ONERA il y a un certain nombre de films assez didactiques sur le décollement toumant qu'on peut se procurer facilement : il suffit de les demander. 